\title{
OPTIMAL CONTROL OF SWITCHED IMPULSIVE SYSTEMS WITH TIME DELAY
}

\author{
K. H. WONG ${ }^{1}$ and W. M. TANG ${ }^{\otimes}$
}

(Received 16 January, 2012; revised 5 September, 2012; first published online 7 February, 2013)

\begin{abstract}
We develop a computational method for solving an optimal control problem governed by a switched impulsive dynamical system with time delay. At each time instant, only one subsystem is active. We propose a computational method for solving this optimal control problem where the time spent by the state in each subsystem is treated as a new parameter. These parameters and the jump strengths of the impulses are decision parameters to be optimized. The gradient formula of the cost function is derived in terms of solving a number of delay differential equations forward in time. Based on this, the optimal control problem can be solved as an optimization problem.
\end{abstract}

2010 Mathematics subject classification: 49J20.

Keywords and phrases: switched and impulsive systems, time delay, subsystems, magnitudes of impulses, duration times, mathematical programming problem.

\section{Introduction}

A hybrid system is a dynamical system that involves both continuous and discrete event dynamics. The continuous dynamics are usually described by differential equations, while the discrete dynamics are described by a switching law. An impulsive hybrid system consists of several subsystems and a switching law, together with an impulsive control law which governs the amount of jump from one subsystem to another. At each time instant, only one subsystem is active. It can be described by a differential inclusion together with an impulsive control law of the form

$$
\begin{aligned}
& \dot{x}(t) \in\left\{f_{v}(t, x(t), u(t)): v \in\{1,2, \ldots, M\}\right\}, \\
& x\left(\tau_{v}^{+}\right)=x\left(\tau_{v}^{-}\right)+g\left(x\left(\tau_{v}^{-}, q_{v}\right)\right),
\end{aligned}
$$

where $x(t) \in \mathbb{R}^{n}$ is the state; $u(t) \in \mathbb{R}^{m}$ is the control;

$$
f_{v}: \mathbb{R}^{n+m+1} \rightarrow \mathbb{R}^{n} \quad \text { and } \quad g: \mathbb{R}^{n} \rightarrow \mathbb{R}^{n} \times \mathbb{R}^{n}
$$

${ }^{1}$ College of Computer and Information Science, Chongqing Normal University, Chongqing 400047, China; e-mail: karwong01@gmail.com, cqtwm@163.com.

(C) Australian Mathematical Society 2013, Serial-fee code 1446-1811/2013 $\$ 16.00$ 
are continuously differentiable with respect to their arguments; $\tau_{v} \in \mathbb{R}$ is the switching time of the state; and $q_{v} \in \mathbb{R}^{n}$ is the amount of jump when the state jumps from one subsystem to another. Optimal control of such a system involves finding an optimal control function $u(t)$ together with optimal switching times $\tau_{v}$ and optimal state jumps $q_{v}$ such that a given cost function is minimized.

Hybrid problems involving switched systems or switched impulsive systems for the delay-free case have been widely studied in the past two decades. For example, a locally optimal feedback control is obtained for the switched impulsive control problem given by Jiang et al. [4] by using a neighbouring extremal solution method. A computational method using the gradient scheme is developed for computing an optimal control of the nonlinear impulsive system given by Lin et al. [7]. (In fact, the gradient scheme proposed in Section 3 of this paper for solving our time-delayed optimal impulsive control problem is similar to that used by Lin et al. [7].) Modelling and optimal control for multistage switched systems with applications to microbial fed-batch culture are dealt with by Liu et al. [8]. We refer also to other relevant articles $[1-3,6,9,10,16]$ and to the relevant references cited therein.

Optimal control problems involving switched systems with time delay but without impulsive control are investigated by Verriest [11, 12] and Wu et al. [14, 15]. However, optimal control problems involving switched impulsive systems with time delay are very rare in the literature, except possibly for a paper by Verriest et al. [13]. In that paper, the optimal control problem is described by a differential inclusion with a timedelay argument in the state variable, but without any time-varying control. In other words, the system is governed by a differential inclusion of the form

$$
\dot{x}(t) \in\left\{f_{v}(t, x(t), x(t-h)): v \in\{1,2, \ldots, M\}\right\}
$$

together with the impulsive control law (1.1). The optimal control problem is cast as a constrained parameter optimization problem with the impulsive condition (1.1) regarded as constraints. The necessary condition for optimality is derived via a classical variational technique. Hence, an optimal control can be found by solving delay differential equations arising from both the state and the adjoint system, together with algebraic equations arising from the necessary condition for optimality as well as the transversality condition due to the impulsive control conditions.

The optimal control problem considered in this paper is almost the same as that considered by Verriest et al. [13]. We propose a computational method for solving this optimal control problem based on the approach reported by Kaya and Noakes [5], Lin et al. [7] and Wu et al. [14]. The time spent by the state in one subsystem is treated as a new parameter $\xi_{i}$ so that the time interval during which the $i$ th subsystem is active can be transformed from $t \in\left[\tau_{i}, \tau_{i+1}\right]$ to $s_{i} \in\left[0, \xi_{i}\right]$. Then the optimal control problem can be easily cast as an optimization problem consisting of a cost function together with some simple bounds on the parameter $\xi_{i}$ only. We derive the gradient formula of the cost function with respect to all the decision parameters, which are the duration times and the state jumps. Since the gradient formula that we obtain depends on the state systems only, the evaluation of both the cost function and its gradient does not involve 
the adjoint system at all. As a consequence, our method is computationally cheaper than that reported by Verriest et al. [13].

The paper is organized as follows. The formulation of the problem is presented in Section 2. We introduce a method to treat the time spent by the state in one subsystem as a new parameter in Section 3. We then derive the gradient formula of the cost function with respect to all the decision parameters in terms of solving a number of delay differential equations forward in time. We solve a numerical example in Section 4 to illustrate the applicability of our proposed method, and some concluding remarks are given in Section 5.

\section{Problem formulation}

Consider the switched impulsive dynamical system defined on $[0, T]$ with one delay and $N$ switches given below:

$$
\dot{x}(t)=f_{i}(x(t), x(t-h)), \quad t \in\left(\tau_{i-1}, \tau_{i}\right), i=1, \ldots, N+1
$$

with impulsive conditions

$$
x\left(\tau_{i}^{+}\right)=x\left(\tau_{i}^{-}\right)+g\left(x\left(\tau_{i}^{-}\right)\right) q_{i}, \quad i=1, \ldots, N
$$

and initial condition

$$
x(0)=x_{0}, \quad x(t)=\phi(t), \quad t \in[-h, 0),
$$

where $x \in \mathbb{R}^{n} ; h$ is the delay time; $f_{i}: \mathbb{R}^{2 n} \rightarrow \mathbb{R}^{n}, i=1, \ldots, N+1, g: \mathbb{R}^{n} \rightarrow \mathbb{R}^{n \times n}$ and $\phi: \mathbb{R}^{1} \rightarrow \mathbb{R}^{n}$ are given functions; $x\left(\tau_{i}^{-}\right)$and $x\left(\tau_{i}^{+}\right)$are, respectively, the state variables just before and after the impulsive control has been applied; and $q_{i} \in \mathbb{R}^{n}$ is the strength of the impulsive control.

We assume that the switching sequence is preassigned, such that

$$
0=\tau_{0} \leq \tau_{1} \leq \cdots \leq \tau_{N} \leq \tau_{N+1}=T \text {. }
$$

Thus, the switching times $\tau_{1}, \ldots, \tau_{N}$ as well as the strengths of the impulsive controls $q_{1}, \ldots, q_{N}$ are decision variables. Our control problem, referred to as Problem (P), is formally stated as follows.

Problem (P). Subject to the switched impulsive system (2.1)-(2.3) and the constraints (2.4), find a switching vector $\tau=\left[\tau_{1}, \ldots, \tau_{N}\right] \in \mathbb{R}^{N}$ and an impulsive control vector $q=\left[q_{1}^{T}, \ldots, q_{N}^{T}\right]^{T} \in \mathbb{R}^{n N}$ such that the cost function

$$
J(\tau, q)=\Phi(x(T \mid \tau, q))
$$

is minimized, where $x(T \mid \tau, q)$ is the solution of (2.1)-(2.3) at the terminal time $T$.

To proceed further, we assume that the following conditions are satisfied. 
(A1) The duration between each pair of consecutive switching times is larger than the delay time $h$. That is,

$$
\tau_{i}-\tau_{i-1} \geq h \quad \text { for all } i=1, \ldots, N+1 .
$$

(A2) The functions $f_{i}(x(t), x(t-h)), i=1, \ldots, N+1, \Phi(x(t)), g(x(t))$ and $\phi(t)$ are continuously differentiable with respect to their arguments.

\section{The model transformation and gradient formula}

Note that the cost function of Problem (P) depends both on the switching vector and the impulsive control vector. Thus, to solve Problem $(\mathrm{P})$, we need to derive the gradient formula of the cost function with respect to the switching vector as well as the impulsive control vector. For this, we first convert each of the time intervals $t \in$ $\left[\tau_{i-1}, \tau_{i}\right], i=1, \ldots, N+1$, into $s_{i} \in\left[0, \xi_{i}\right], i=1, \ldots, N+1$, in the following manner.

Let

$$
\xi_{i}=\tau_{i}-\tau_{i-1}, \quad i=1, \ldots, N+1,
$$

be the duration between the switching times $\tau_{i-1}$ and $\tau_{i}$. Let $\xi=\left(\xi_{1}, \ldots, \xi_{N+1}\right) \in \mathbb{R}^{N+1}$ be the duration vector. From (3.1) and (2.5),

$$
\begin{aligned}
& \xi_{i} \geq h, \quad i=1, \ldots, N+1, \\
& \sum_{i=1}^{j} \xi_{i}=\tau_{j}, \\
& \sum_{i=1}^{N+1} \xi_{i}=T .
\end{aligned}
$$

Set

$$
s_{i}=t-\tau_{i-1}, \quad t \in\left[\tau_{i-1}, \tau_{i}\right], i=1, \ldots, N+1,
$$

so that $s_{i} \in\left[0, \xi_{i}\right]$ whenever $t \in\left[\tau_{i-1}, \tau_{i}\right]$. Furthermore, for each $i=1, \ldots, N+1$ define

$$
\begin{aligned}
& \hat{x}_{i}\left(s_{i}\right)=x\left(s_{i}+\tau_{i-1}\right), \quad s_{i} \in\left(0, \xi_{i}\right), \\
& \hat{x}_{i}(0)=x\left(\tau_{i-1}^{+}\right), \\
& \hat{x}_{i}\left(\xi_{i}\right)=x\left(\tau_{i}^{-}\right),
\end{aligned}
$$

with

$$
\begin{aligned}
& \hat{x}_{i}\left(s_{i}-h\right)=\hat{x}_{i-1}\left(\xi_{i}+s_{i}-h\right), \quad s_{i} \in[0, h), i=2, \ldots, N, \\
& \hat{x}_{1}\left(s_{1}-h\right)=\phi\left(s_{1}-h\right), \quad s_{1} \in[0, h) .
\end{aligned}
$$

Thus, equations (2.1) and (2.2) become

$$
\begin{array}{ll}
\dot{\hat{x}}_{i}\left(s_{i}\right)=f_{i}\left(\hat{x}_{i}\left(s_{i}\right), \hat{x}_{i}\left(s_{i}-h\right)\right), & s_{i} \in\left(0, \xi_{i}\right), i=2, \ldots, N+1, \\
\hat{x}_{i+1}(0)=\hat{x}_{i}\left(\xi_{i}\right)+g\left(\hat{x}_{i}\left(\xi_{i}\right)\right) q_{i}, & i=1, \ldots, N .
\end{array}
$$


We see that $\hat{x}_{i+1}\left(s_{i+1}\right)$ depends on the duration vector as well as the impulsive control vector during each of the time intervals $s_{i+1} \in\left(0, \xi_{i+1}\right), i=1, \ldots, N$. That is, for $i=1, \ldots, N$,

$$
\hat{x}_{i+1}\left(s_{i+1}\right)=\hat{x}_{i+1}\left(s_{i+1} ; \xi_{i}, \ldots, \xi_{1}, q_{i}, \ldots, q_{1}\right)
$$

whenever $s_{i+1} \in\left(0, \xi_{i+1}\right)$. Thus, (3.4) can be written as

$$
\begin{aligned}
& \frac{\partial \hat{x}_{i+1}\left(s_{i+1} ; \xi_{i}, \ldots, \xi_{1}, q_{i}, \ldots, q_{1}\right)}{\partial s_{i+1}} \\
& =f_{i+1}\left(\hat{x}_{i+1}\left(s_{i+1} ; \xi_{i}, \ldots, \xi_{1}, q_{i}, \ldots, q_{1}\right), \hat{x}_{i+1}\left(s_{i+1}-h ; \xi_{i}, \ldots, \xi_{1}, q_{i}, \ldots, q_{1}\right)\right) \\
& \quad s_{i+1} \in\left(0, \xi_{i+1}\right), \quad i=1, \ldots, N
\end{aligned}
$$

and

$$
\frac{d \hat{x}_{1}}{d s_{1}}=f_{1}\left(\hat{x}_{1}\left(s_{1}\right), \hat{x}_{1}\left(s_{1}-h\right)\right), \quad s_{1} \in\left(0, \xi_{1}\right) .
$$

Moreover, (3.5) can be written as

$$
\begin{aligned}
\hat{x}_{i+1}\left(s_{i+1}\right. & \left.; \xi_{i}, \ldots, \xi_{1}, q_{i}, \ldots, q_{1}\right)\left.\right|_{s_{i+1}=0} \\
\quad= & \left.\hat{x}_{i}\left(s_{i} ; \xi_{i-1}, \ldots, \xi_{1}, q_{i-1}, \ldots, q_{1}\right)\right|_{s_{i}=\xi_{i}} \\
& \quad+\left.g\left(\hat{x}_{i}\left(s_{i} ; \xi_{i-1}, \ldots, \xi_{1}, q_{i-1}, \ldots, q_{1}\right)\right)\right|_{s_{i}=\xi_{i}} q_{i}, \quad i=1, \ldots, N .
\end{aligned}
$$

The initial condition (2.3) becomes

$$
\left.\hat{x}_{1}\left(s_{1}\right)\right|_{s_{1}=0}=x_{0}, \quad \hat{x}_{1}\left(s_{1}-h\right)=\phi\left(s_{1}-h\right), \quad s_{1} \in[-h, 0) .
$$

Write

$$
J(\xi, q)=\Phi\left(\hat{x}_{N+1}\left(\xi_{N+1} \mid \xi_{N}, \ldots, \xi_{1}, q_{N}, \ldots, q_{1}\right)\right),
$$

where $\hat{x}_{N+1}\left(\cdot \mid \xi_{N}, \ldots, \xi_{1}, q_{N}, \ldots, q_{1}\right)$ is the solution of (3.6)-(3.9) corresponding to the parameters $\xi$ and $q$. Now Problem (P) can be stated equivalently as Problem (RP) given below.

Problem (RP). Given the dynamical system (3.6)-(3.9), find a duration vector $\xi \in$ $\mathbb{R}^{N+1}$ and an impulsive control vector $q \in \mathbb{R}^{n N}$ such that (3.10) is minimized subject to (3.2) and (3.3).

To develop an optimization method to solve Problem (RP), we need to derive the gradient formula of the cost function (3.10) with respect to the duration vector $\xi$ and the impulsive control vector $q$. We introduce the following notation. For any function $G: \mathbb{R}^{m} \rightarrow \mathbb{R}^{n}$, define

$$
\frac{\partial G}{\partial x}=\left(\begin{array}{ccc}
\frac{\partial G_{1}}{\partial x_{1}} & \cdots & \frac{\partial G_{1}}{x_{n}} \\
\vdots & \ddots & \vdots \\
\frac{\partial G_{m}}{\partial x_{1}} & \cdots & \frac{\partial G_{m}}{x_{n}}
\end{array}\right)
$$


Then

$$
\begin{aligned}
\frac{\partial J(\xi, q)}{\partial \xi_{i}} & =\frac{\partial \Phi\left(\hat{x}_{N+1}\left(\xi_{N+1} \mid \xi, q\right)\right)}{\partial \xi_{i}} \\
& =\frac{\partial \Phi\left(\hat{x}_{N+1}\left(\xi_{N+1} \mid \xi, q\right)\right)}{\partial \hat{x}_{N+1}} \frac{\partial \hat{x}_{N+1}\left(\xi_{N+1} \mid \xi, q\right)}{\partial \xi_{i}} \\
\frac{\partial J(\xi, q)}{\partial q_{i}} & =\frac{\partial \Phi\left(\hat{x}_{N+1}\left(\xi_{N+1} \mid \xi, q\right)\right)}{\partial q_{i}} \\
& =\frac{\partial \Phi\left(\hat{x}_{N+1}\left(\xi_{N+1} \mid \xi, q\right)\right)}{\partial \hat{x}_{N+1}} \frac{\partial \hat{x}_{N+1}\left(\xi_{N+1} \mid \xi, q\right)}{\partial q_{i}} .
\end{aligned}
$$

(Note that $\partial J(\xi, q) / \partial \xi_{i} \in \mathbb{R}$ and $\partial J(\xi, q) / \partial q_{i} \in \mathbb{R}^{n}$.) We need to find

$$
\frac{\partial x_{N+1}\left(\xi_{N+1} \mid \xi, q\right)}{\partial \xi_{1}}, \ldots, \frac{\partial x_{N+1}\left(\xi_{N+1} \mid \xi, q\right)}{\partial \xi_{N+1}}, \frac{\partial x_{N+1}\left(\xi_{N+1} \mid \xi, q\right)}{\partial q_{1}}, \ldots, \frac{\partial x_{N+1}\left(\xi_{N+1} \mid \xi, q\right)}{\partial q_{N}}
$$

Theorem 3.1. Define $\tilde{x}_{i}\left(s_{i}\right)=\hat{x}_{i}\left(s_{i}-h\right)$ and let $y_{(1)}^{(i, i+j)}\left(s_{j}\right) \in \mathbb{R}^{n}, y_{(2)}^{(i, i+j)}\left(s_{j}\right) \in \mathbb{R}^{n} \times \mathbb{R}^{n}$, $i=1, \ldots, N, j=1, \ldots, N-i+1$. Furthermore, let $y_{(1)}^{(i, i+j)}\left(s_{j}\right)$ and $y_{(2)}^{(i, i+j)}\left(s_{j}\right)$ be, respectively, the solutions of the vector-valued delay differential equation and matrixvalued delay differential equation given by

$$
\begin{aligned}
\frac{d y_{(k)}^{(i, i+1)}\left(s_{i+1}\right)}{d s_{i+1}}= & \frac{\partial f_{i+1}\left(\hat{x}_{i+1}\left(s_{i+1}\right), \tilde{x}_{i+1}\left(s_{i+1}\right)\right)}{\partial \hat{x}_{i+1}} y_{(k)}^{(i, i+1)}\left(s_{i+1}\right) \\
& +\frac{\partial f_{i+1}\left(\hat{x}_{i+1}\left(s_{i+1}\right), \tilde{x}_{i+1}\left(s_{i+1}\right)\right)}{\partial \tilde{x}_{i+1}} \tilde{y}_{(k)}^{(i, i+1)}\left(s_{i+1}\right), \\
\frac{d y_{(k)}^{(i, i+2)}\left(s_{i+2}\right)}{d s_{i+2}}= & \frac{\partial f_{i+2}\left(\hat{x}_{i+2}\left(s_{i+2}\right), \tilde{x}_{i+2}\left(s_{i+2}\right)\right)}{\partial \hat{x}_{i+2}} y_{(k)}^{(i, i+2)}\left(s_{i+2}\right) \\
& +\frac{\partial f_{i+2}\left(\hat{x}_{i+2}\left(s_{i+2}\right), \tilde{x}_{i+2}\left(s_{i+2}\right)\right)}{\partial \tilde{x}_{i+2}} \tilde{y}_{(k)}^{(i, i+2)}\left(s_{i+2}\right), \\
\frac{d y_{(k)}^{(i, N+1)}\left(s_{N+1}\right)}{d s_{N+1}}= & \frac{\partial f_{N+1}\left(\hat{x}_{N+1}\left(s_{N+1}\right), \tilde{x}_{N+1}\left(s_{N+1}\right)\right)}{\partial \hat{x}_{N+1}} y_{(k)}^{(i, N+1)}\left(s_{N+1}\right) \\
& +\frac{\partial f_{N+1}\left(\hat{x}_{N+1}\left(s_{N+1}\right), \tilde{x}_{N+1}\left(s_{N+1}\right)\right)}{\partial \tilde{x}_{N+1}} \tilde{y}_{(k)}^{(i, N+1)}\left(s_{N+1}\right),
\end{aligned}
$$


where $k=1,2, s_{i+1} \in\left(0, \xi_{i+1}\right), s_{i+2} \in\left(0, \xi_{i+2}\right), \ldots, s_{N+1} \in\left(0, \xi_{N+1}\right)$, with initial conditions for $y_{(1)}^{(i, i+1)}\left(s_{i+1}\right), \ldots, y_{(1)}^{(i, N+1)}\left(s_{N+1}\right)$ given by

$$
\begin{gathered}
\left.y_{(1)}^{(i, i+1)}\left(s_{i+1}\right)\right|_{s_{i+1}=0}=\left[I+\frac{\partial g\left(\hat{x}_{i}\left(\xi_{i}\right)\right) q_{i}}{\partial \hat{x}_{i}}\right] f_{i}\left(\hat{x}_{i}\left(\xi_{i}\right), \tilde{x}\left(\xi_{i}\right)\right), \\
\left.y_{(1)}^{(i, i+j)}\left(s_{i+j}\right)\right|_{s_{i+j}=0}=\left.\left[I+\frac{\partial g\left(\hat{x}_{i+j-1}\left(\xi_{i+j-1}\right)\right) q_{i+j-1}}{\partial \hat{x}_{i+j-1}}\right] y_{(1)}^{(i, i+j-1)}\left(s_{i+j-1}\right)\right|_{s_{i+j-1}=\xi_{i+j-1}}, \\
j=2, \ldots, N-i+1,
\end{gathered}
$$

and initial conditions for $y_{(2)}^{(i, i+1)}\left(s_{i+1}\right), \ldots, y_{(2)}^{(i, N+1)}\left(s_{N+1}\right)$ given by

$$
\begin{gathered}
\left.y_{(2)}^{(i, i+1)}\left(s_{i+1}\right)\right|_{s_{i+1}=0}=g\left(\hat{x}_{i}\left(\xi_{i}\right)\right), \\
\left.y_{(2)}^{(i, i+j)}\left(s_{i+j}\right)\right|_{s_{i+j}=0}=\left.\left[I+\frac{\partial g\left(\hat{x}_{i+j-1}\left(\xi_{i+j-1}\right) q_{i+j-1}\right)}{\partial \hat{x}_{i+j-1}}\right] y_{(2)}^{(i, i+j-1)}\left(s_{i+j-1}\right)\right|_{s_{i+j-1}=\xi_{i+j-1}}, \\
j=2, \ldots, N-i+1,
\end{gathered}
$$

where

$$
\begin{aligned}
& \tilde{y}_{(k)}^{(i, i+j)}\left(s_{i+j}\right)=y_{(k)}^{(i, i+j)}\left(s_{i+j}-h\right), \\
& \quad i=1, \ldots, N, j=1, \ldots, N-i+1, k=1,2, s_{i+j} \in\left(h, \xi_{i+j}\right], \\
& \tilde{y}_{(k)}^{(i, i+j)}\left(s_{i+j}\right)=\tilde{0}, \\
& \quad i=1, \ldots, N, j=1, \ldots, N-i+1, k=1,2, s_{i+j} \in[0, h],
\end{aligned}
$$

and $\tilde{0}$ is the zero vector in $\mathbb{R}^{n}$. Then

$$
\begin{aligned}
& \frac{\partial \hat{x}_{N+1}\left(\xi_{N+1} \mid \xi, q\right)}{\partial \xi_{1}}=y_{(1)}^{(1, N+1)}\left(\xi_{N+1}\right), \ldots, \frac{\partial \hat{x}_{N+1}\left(\xi_{N+1} \mid \xi, q\right)}{\partial \xi_{N}}=y_{(1)}^{(N, N+1)}\left(\xi_{N+1}\right), \\
& \frac{\partial \hat{x}_{N+1}\left(\xi_{N+1} \mid \xi, q\right)}{\partial \xi_{N+1}}=f_{N+1}\left(\hat{x}_{N+1}\left(\xi_{N+1}\right), \tilde{x}_{N+1}\left(\xi_{N+1}\right)\right), \\
& \frac{\partial \hat{x}_{N+1}\left(\xi_{N+1} \mid \xi, q\right)}{\partial q_{1}}=y_{(2)}^{(1, N+1)}\left(\xi_{N+1}\right), \ldots, \frac{\partial \hat{x}_{N+1}\left(\xi_{N+1} \mid \xi, q\right)}{\partial q_{N}}=y_{(2)}^{(N, N+1)}\left(\xi_{N+1}\right) .
\end{aligned}
$$

Proof. Since $f_{i}\left(\hat{x}\left(s_{i}\right), \tilde{x}\left(s_{i}\right)\right), i=1, \ldots, N+1$, are continuously differentiable with respect to their arguments, it follows that for each $i=1, \ldots, N+1$ we can use equation (3.6) to obtain the partial derivatives

$$
\begin{gathered}
\frac{\partial^{2} \hat{x}_{i+1}\left(s_{i+1} \mid \xi_{i}, \ldots, \xi_{1}, q_{i}, \ldots, q_{1}\right)}{\partial \xi_{i} \partial s_{i+1}}, \frac{\partial^{2} \hat{x}_{i+2}\left(s_{i+2} \mid \xi_{i+1}, \ldots, \xi_{1}, q_{i+1}, \ldots, q_{1}\right)}{\partial \xi_{i} \partial s_{i+2}}, \\
\ldots, \frac{\partial^{2} \hat{x}_{N+1}\left(s_{N+1} \mid \xi_{N}, \ldots, \xi_{1}, q_{N}, \ldots, q_{1}\right)}{\partial \xi_{i} \partial s_{N+1}}
\end{gathered}
$$


as follows:

$$
\begin{aligned}
& \frac{\partial^{2} \hat{x}_{i+j}\left(s_{i+j} ; \xi_{i+j-1}, \ldots, \xi_{1}, q_{i+j-1}, \ldots, q_{1}\right)}{\partial \xi_{i} \partial s_{i+j}} \\
= & \frac{\partial^{2} \hat{x}_{i+j}\left(s_{i+j} ; \xi_{i+j-1}, \ldots, \xi_{1}, q_{i+j-1}, \ldots, q_{1}\right)}{\partial s_{i+j} \partial \xi_{i}} \\
= & \frac{\partial f_{i+j}\left(\hat{x}_{i+j}\left(s_{i+j} ; \xi_{i+j-1}, \ldots, \xi_{1}, q_{i+j-1}, \ldots, q_{1}\right), \tilde{x}_{i+j}\left(s_{i+j} ; \xi_{i+j-1}, \ldots, \xi_{1}, q_{i+j-1}, \ldots, q_{1}\right)\right)}{\partial \xi_{i}} \\
= & \frac{\partial f_{i+j}\left(\hat{x}_{i+j}\left(s_{i+j} ; \xi_{i+j-1}, \ldots, \xi_{1}, q_{i+j-1}, \ldots, q_{1}\right), \tilde{x}_{i+j}\left(s_{i+j} ; \xi_{i+j-1}, \ldots, \xi_{1}, q_{i+j-1}, \ldots, q_{1}\right)\right)}{\partial \hat{x}_{i+j}} \\
& \times \frac{\partial \hat{x}_{i+j}\left(s_{i+j} ; \xi_{i+j-1}, \ldots, \xi_{1}, q_{i+j-1}, \ldots, q_{1}\right)}{\partial \xi_{i}} \\
& +\frac{\partial f_{i+j}\left(\tilde{x}_{i+j}\left(s_{i+j} ; \xi_{i+j-1}, \ldots, \xi_{1}, q_{i+j-1}, \ldots, q_{1}\right), \tilde{x}_{i+j}\left(s_{i+j} ; \xi_{i+j-1}, \ldots, \xi_{1}, q_{i+j-1}, \ldots, q_{1}\right)\right)}{\partial \tilde{x}_{i}} \\
& \times \frac{\partial \tilde{x}_{i+j}\left(s_{i+j} ; \xi_{i+j-1}, \ldots, \xi_{1}, q_{i+j-1}, \ldots, q_{1}\right)}{\partial \xi_{i}}, \quad i=1, \ldots, N, j=1, \ldots, N-i+1,
\end{aligned}
$$

for all $s_{i+j} \in\left(0, \xi_{i+j}\right)$. Since $\hat{x}_{i}\left(s_{i}\right), \tilde{x}_{i}\left(s_{i}\right)$ and $f_{i}\left(\hat{x}_{i}\left(s_{i}\right), \tilde{x}_{i}\left(s_{i}\right)\right)$ are continuous with respect to their arguments, it follows from (3.6) and (3.8) that the initial conditions for

$$
\begin{gathered}
\frac{\partial \hat{x}_{i+1}\left(s_{i+1} \mid \xi_{i}, \ldots, \xi_{1}, q_{i}, \ldots, q_{1}\right)}{\partial \xi_{i}}, \frac{\partial \hat{x}_{i+2}\left(s_{i+2} \mid \xi_{i+1}, \ldots, \xi_{1}, q_{i+1}, \ldots, q_{1}\right)}{\partial \xi_{i}}, \\
\ldots, \frac{\partial \hat{x}_{N+1}\left(s_{N+1} \mid \xi_{N}, \ldots, \xi_{1}, q_{N}, \ldots, q_{1}\right)}{\partial \xi_{i}}
\end{gathered}
$$

are obtained as follows:

$$
\begin{aligned}
&\left.\frac{\partial \hat{x}_{i+1}\left(s_{i+1} ; \xi_{i}, \ldots, \xi_{1}, q_{i}, \ldots, q_{1}\right)}{\partial \xi_{i}}\right|_{s_{i+1}=0} {\left.\left[I+\frac{\partial g\left(\hat{x}_{i}\left(\xi_{i}\right)\right) q_{i}}{\partial \hat{x}_{i}}\right] \frac{\partial \hat{x}_{i}\left(s_{i} ; \xi_{i-1}, \ldots, \xi_{1}, q_{i-1}, \ldots, q_{1}\right)}{\partial s_{i}}\right|_{s_{i}=\xi_{i}^{-}} } \\
&= {\left[I+\frac{\partial g\left(\hat{x}_{i}\left(\xi_{i}\right)\right) q_{i}}{\partial \hat{x}_{i}}\right] f_{i}\left(\hat{x}\left(\xi_{i}^{-}\right), \tilde{x}\left(\xi_{i}^{-}\right)\right) } \\
&= {\left[I+\frac{\partial g\left(\hat{x}_{i}\left(\xi_{i}\right)\right) q_{i}}{\partial \hat{x}_{i}}\right] f_{i}\left(\hat{x}\left(\xi_{i}\right), \tilde{x}\left(\xi_{i}\right)\right), \quad i=1, \ldots, N, } \\
&\left.\frac{\partial \hat{x}_{i+j}\left(s_{i+j} ; \xi_{i+j-1}, \ldots, \xi_{i}, \ldots, \xi_{1}, q_{i+j-1}, \ldots, q_{i}, \ldots, q_{1}\right)}{\partial \xi_{i}}\right|_{s_{i+j}=0} \\
&=\left[I+\frac{\partial g\left(\hat{x}_{i+j-1}\left(\xi_{i+j-1}\right)\right) q_{i+j-1}}{\partial \hat{x}_{i+j-1}}\right] \\
& \quad \times\left.\frac{\partial \hat{x}_{i+j-1}\left(s_{i+j-1} ; \xi_{i+j-1}, \ldots, \xi_{1}, q_{i+j-1}, \ldots, q_{1}\right)}{\partial \xi_{i}}\right|_{s_{i+j-1}=\xi_{i+j-1}}, \\
& \quad i=1, \ldots, N-1, j=2, \ldots, N-i+1 .
\end{aligned}
$$


Similarly, for each $i=1, \ldots, N+1$, we use equation (3.6) to obtain the partial derivatives

$$
\begin{gathered}
\frac{\partial^{2} \hat{x}_{i+1}\left(s_{i+1} \mid \xi_{i}, \ldots, \xi_{1}, q_{i}, \ldots, q_{1}\right)}{\partial q_{i} \partial s_{i+1}}, \frac{\partial^{2} \hat{x}_{i+2}\left(s_{i+2} \mid \xi_{i+1}, \ldots, \xi_{1}, q_{i+1}, \ldots, q_{1}\right)}{\partial q_{i} \partial s_{i+2}}, \\
\ldots, \frac{\partial^{2} \hat{x}_{N+1}\left(s_{N+1} \mid \xi_{N}, \ldots, \xi_{1}, q_{N}, \ldots, q_{1}\right)}{\partial q_{i} \partial s_{N+1}}
\end{gathered}
$$

(Note that $\partial^{2} \hat{x}_{i+1} / \partial q_{i} \partial s_{i+1}, \partial^{2} \hat{x}_{i+2} / \partial q_{i} \partial s_{i+2}, \ldots, \partial^{2} \hat{x}_{N+1} / \partial q_{i} \partial s_{N+1}$ are all $n \times n$ matrices because $q_{i} \in \mathbb{R}^{n}$.) They are

$$
\begin{aligned}
& \frac{\partial^{2} \hat{x}_{i+j}\left(s_{i+j} ; \xi_{i+j-1}, \ldots, \xi_{1}, q_{i+j-1}, \ldots, q_{1}\right)}{\partial q_{i} \partial s_{i+j}} \\
= & \frac{\partial^{2} \hat{x}_{i+j}\left(s_{i+j} ; \xi_{i+j-1}, \ldots, \xi_{1}, q_{i+j-1}, \ldots, q_{1}\right)}{\partial s_{i+j} \partial q_{i}} \\
= & \frac{\partial f_{i+j}\left(\hat{x}_{i+j}\left(s_{i+j} ; \xi_{i+j-1}, \ldots, \xi_{1}, q_{i+j-1}, \ldots, q_{1}\right), \tilde{x}_{i+j}\left(s_{i+j} ; \xi_{i+j-1}, \ldots, \xi_{1}, q_{i+j-1}, \ldots, q_{1}\right)\right)}{\partial q_{i}} \\
= & \frac{\partial f_{i+j}\left(\hat{x}_{i+j}\left(s_{i+j} ; \xi_{i+j-1}, \ldots, \xi_{1}, q_{i+j-1}, \ldots, q_{1}\right), \tilde{x}_{i+j}\left(s_{i+j} ; \xi_{i+j-1}, \ldots, \xi_{1}, q_{i+j-1}, \ldots, q_{1}\right)\right)}{\partial \hat{x}_{i+j}} \\
& \times \frac{\partial \hat{x}_{i+j}\left(s_{i+j} ; \xi_{i+j-1}, \ldots, \xi_{1}, q_{i+j-1}, \ldots, q_{1}\right)}{\partial q_{i}} \\
& +\frac{\partial f_{i+j}\left(\tilde{x}_{i+j}\left(s_{i+j} ; \xi_{i+j-1}, \ldots, \xi_{1}, q_{i+j-1}, \ldots, q_{1}\right), \tilde{x}_{i+j}\left(s_{i+j} ; \xi_{i+j-1}, \ldots, \xi_{1}, q_{i+j-1}, \ldots, q_{1}\right)\right)}{\partial \tilde{x}_{i+j}} \\
& \times \frac{\partial \tilde{x}_{i+j}\left(s_{i+j} ; \xi_{i+j-1}, \ldots, \xi_{1}, q_{i+j-1}, \ldots, q_{1}\right)}{\partial q_{i}}, \quad i=1, \ldots, N, j=1, \ldots, N-i+1,
\end{aligned}
$$

for all $s_{i+j} \in\left(0, \xi_{i+j}\right)$. It follows from (3.8) and (3.6) that the initial conditions for

$$
\begin{gathered}
\frac{\partial \hat{x}_{i+1}\left(s_{i+1} \mid \xi_{i}, \ldots, \xi_{1}, q_{i}, \ldots, q_{1}\right)}{\partial q_{i}}, \frac{\partial \hat{x}_{i+2}\left(s_{i+2} \mid \xi_{i+1}, \ldots, \xi_{1}, q_{i+1}, \ldots, q_{1}\right)}{\partial q_{i}}, \\
\ldots, \frac{\partial \hat{x}_{N+1}\left(s_{N+1} \mid \xi_{N}, \ldots, \xi_{1}, q_{N}, \ldots, q_{1}\right)}{\partial q_{i}}
\end{gathered}
$$

are as follows:

$$
\left.\frac{\partial \hat{x}_{i+1}\left(s_{i+1} \mid \xi_{i}, \ldots, \xi_{1}, q_{i}, \ldots, q_{1}\right)}{\partial q_{i}}\right|_{s_{i+1}=0}=g\left(\hat{x}_{i}\left(\xi_{i}\right)\right), \quad i=1, \ldots, N,
$$

and

$$
\begin{aligned}
\left.\frac{\partial \hat{x}_{i+j}\left(s_{i+j} \mid \xi_{i+j-1}, \ldots, \xi_{i}, \ldots, \xi_{1}, q_{i+j-1}, \ldots, q_{i}, \ldots, q_{1}\right)}{\partial q_{i}}\right|_{s_{i+j}=0} \\
=\left[I+\frac{\partial g\left(\hat{x}_{i+j-1}\left(\xi_{i+j-1}\right)\right) q_{i+j-1}}{\partial \hat{x}_{i+j-1}}\right] \\
\quad \times\left.\frac{\partial \hat{x}_{i+j-1}\left(s_{i+j-1} ; \xi_{i+j-1}, \ldots, \xi_{1}, q_{i+j-1}, \ldots, q_{1}\right)}{\partial q_{i}}\right|_{s_{i+j-1}=\xi_{i+j-1}}, \\
\quad i=1, \ldots, N-1, j=2, \ldots, N-i+1 .
\end{aligned}
$$


The conclusion of the theorem follows by letting

$$
\begin{aligned}
y_{(1)}^{(i, i+j)}\left(s_{j}\right) & =\frac{\partial \hat{x}_{i+j}\left(s_{i+j} ; \xi_{i+j-1}, \ldots, \xi_{1}, q_{i+j-1}, \ldots, q_{1}\right)}{\partial \xi_{i}}, \\
y_{(2)}^{(i, i+j)}\left(s_{j}\right) & =\frac{\partial \hat{x}_{i+j}\left(s_{i+j} ; \xi_{i+j-1}, \ldots, \xi_{1}, q_{i+j-1}, \ldots, q_{1}\right)}{\partial q_{i}},
\end{aligned}
$$

for $i=1, \ldots, N, j=1, \ldots, N-i+1$.

Based on Theorem 3.1, we are in a position to develop an algorithm for solving Problem (RP) as follows.

Algorithm 1.

Step 0. Choose $\xi=\left(\xi_{1}, \ldots, \xi_{N+1}\right) \in \mathbb{R}^{N+1}$ and $q=\left(q_{1}, \ldots, q_{N}\right) \in \mathbb{R}^{N}$ such that (3.2) and (3.3) are satisfied.

Step 1. Solve the delay differential equations (3.6) and (3.7) with initial condition (3.9) and impulsive condition (3.8), yielding $x_{1}\left(s_{1}\right), s_{1} \in\left(0, \xi_{1}\right)$, and $x_{i+1}\left(s_{i+1} ; \xi_{1}, \ldots, \xi_{i}, q_{1}, \ldots, q_{i}\right)$ on each of the time intervals $s_{i+1} \in\left(0, \xi_{i+1}\right)$ for $i=1, \ldots, N$.

Step 2. Let $k=1$. Solve the delay differential equations given by (3.13)-(3.15) with initial conditions (3.16) and (3.17), yielding

$$
\frac{\partial \hat{x}_{N+1}\left(\xi_{N+1} \mid \xi, q\right)}{\partial \xi_{1}}, \frac{\partial \hat{x}_{N+1}\left(\xi_{N+1} \mid \xi, q\right)}{\partial \xi_{2}}, \ldots, \frac{\partial \hat{x}_{N+1}\left(\xi_{N+1} \mid \xi, q\right)}{\partial \xi_{N}}
$$

from (3.20). Furthermore, by (3.21) and step 1, compute

$$
\frac{\partial \hat{x}_{N+1}\left(\xi_{N+1} \mid \xi, q\right)}{\partial \xi_{N+1}}
$$

Step 3. Let $k=2$. Solve the delay differential equations given by (3.13)-(3.15) with initial conditions (3.18) and (3.19), yielding

$$
\frac{\partial \hat{x}_{N+1}\left(\xi_{N+1} \mid \xi, q\right)}{\partial q_{1}}, \frac{\partial \hat{x}_{N+1}\left(\xi_{N+1} \mid \xi, q\right)}{\partial q_{2}}, \ldots, \frac{\partial \hat{x}_{N+1}\left(\xi_{N+1} \mid \xi, q\right)}{\partial q_{N}}
$$

from (3.22).

Step 4. By virtue of (3.11), the gradients of the cost function with respect to $\xi_{i}$, $i=1, \ldots, N+1$, namely

$$
\frac{\partial J(\xi, q)}{\partial \xi_{i}}, \quad i=1, \ldots, N+1,
$$

are computed. By virtue of (3.12), the gradients of the cost function with respect to $q_{i}, i=1, \ldots, N+1$, namely

$$
\frac{\partial J(\xi, q)}{\partial q_{i}}, \quad i=1, \ldots, N
$$

are computed.

Step 5. Solve Problem (RP) as an optimization problem by sequential quadratic programming approximation with active set strategy. 


\section{An illustrative example}

Consider

$$
\begin{aligned}
& \left\{\begin{array}{l}
\dot{x}_{1}(t)=2 x_{1}(t) x_{2}(t)+x_{2}(t-0.1), \\
\dot{x}_{2}(t)=3 x_{1}(t)+4 x_{2}(t-0.1),
\end{array} \quad t \in\left(0, \tau_{1}\right),\right. \\
& \left\{\begin{array}{l}
\dot{x}_{1}(t)=-2 x_{1}(t) x_{2}(t)+\sin \left(x_{2}(t-0.1)\right), \\
\dot{x}_{2}(t)=x_{1}(t) x_{2}(t)+x_{1}(t-0.1) x_{2}(t-0.1),
\end{array} \quad t \in\left(\tau_{1}, \tau_{2}\right),\right. \\
& \left\{\begin{array}{l}
\dot{x}_{1}(t)=-2 x_{1}(t)+3 x_{2}(t-0.1), \\
\dot{x}_{2}(t)=-x_{2}(t)+x_{1}(t-0.1) x_{2}(t-0.1),
\end{array} \quad t \in\left(\tau_{2}, 1\right),\right.
\end{aligned}
$$

with initial condition

$$
x_{1}(t)=-1, \quad x_{2}(t)=t^{2}+1, \quad t \in[-0.1,0],
$$

and impulsive condition

$$
x\left(\tau_{i}^{+}\right)=x\left(\tau_{i}^{-}\right)+g\left(x\left(\tau_{i}^{-}\right)\right) q_{i}, \quad i=1,2,
$$

where

$$
g\left(x\left(\tau_{i}^{-}\right)\right)=\left(\begin{array}{cc}
x_{2}\left(\tau_{i}^{-}\right) & 0 \\
0 & x_{1}\left(\tau_{i}^{-}\right)
\end{array}\right)
$$

The cost function is

$$
J(\tau, q)=\left(x_{1}(1 \mid \tau, q)-0.5\right)^{2}+\left(x_{2}(1 \mid \tau, q)-0.25\right)^{2} .
$$

Using the model transformation method described in Section 3 with exactly the same notation as that defined in that section, the new system becomes:

$$
\begin{aligned}
& \left\{\begin{array}{l}
\dot{\hat{x}}_{1,1}\left(s_{1}\right)=2 \hat{x}_{1,1}\left(s_{1}\right) \hat{x}_{1,2}\left(s_{1}\right)+\tilde{x}_{1,2}\left(s_{1}\right), \\
\dot{\hat{x}}_{1,2}\left(s_{1}\right)=3 \hat{x}_{1,1}\left(s_{1}\right)+4 \tilde{x}_{1,2}\left(s_{1}\right),
\end{array}\right. \\
& \left\{\begin{array}{l}
\dot{\hat{x}}_{2,1}\left(s_{2}\right)=-2 \hat{x}_{2,1}\left(s_{2}\right) \hat{x}_{2,2}\left(s_{2}\right)+\sin \left(\tilde{x}_{2,2}\left(s_{2}\right)\right), \\
\dot{\hat{x}}_{2,2}\left(s_{2}\right)=\hat{x}_{2,1}\left(s_{2}\right) \hat{x}_{2,2}\left(s_{2}\right)+\tilde{x}_{2,1}\left(s_{2}\right) \tilde{x}_{2,2}\left(s_{2}\right),
\end{array} \quad s_{2} \in\left(0, \xi_{2}\right),\right. \\
& \left\{\begin{array}{l}
\dot{\hat{x}}_{3,1}\left(s_{3}\right)=-2 \hat{x}_{3,1}\left(s_{3}\right)+3 \tilde{x}_{3,2}\left(s_{3}\right), \\
\hat{\hat{x}}_{3,2}\left(s_{3}\right)=-\hat{x}_{3,2}\left(s_{3}\right)+\tilde{x}_{3,1}\left(s_{3}\right) \tilde{x}_{3,2}\left(s_{3}\right),
\end{array} \quad s_{3} \in\left(0, \xi_{3}\right),\right.
\end{aligned}
$$

with initial condition

$$
\hat{x}_{1,1}\left(s_{1}\right)=-1, \quad \hat{x}_{1,2}\left(s_{1}\right)=s_{1}^{2}+1, \quad s_{1} \in[-0.1,0],
$$

and impulsive conditions

$$
\begin{aligned}
\left(\begin{array}{l}
\hat{x}_{2,1}(0) \\
\hat{x}_{2,2}(0)
\end{array}\right) & =\left(\begin{array}{l}
\hat{x}_{1,1}\left(\xi_{1}\right) \\
\hat{x}_{1,2}\left(\xi_{1}\right)
\end{array}\right)+\left(\begin{array}{cc}
\hat{x}_{1,2}\left(\xi_{1}\right) & 0 \\
0 & \hat{x}_{1,1}\left(\xi_{1}\right)
\end{array}\right)\left(\begin{array}{l}
q_{1,1} \\
q_{1,2}
\end{array}\right), \\
\left(\begin{array}{l}
\hat{x}_{3,1}(0) \\
\hat{x}_{3,2}(0)
\end{array}\right) & =\left(\begin{array}{l}
\hat{x}_{2,1}\left(\xi_{2}\right) \\
\hat{x}_{2,2}\left(\xi_{2}\right)
\end{array}\right)+\left(\begin{array}{cc}
\hat{x}_{2,2}\left(\xi_{2}\right) & 0 \\
0 & \hat{x}_{2,1}\left(\xi_{2}\right)
\end{array}\right)\left(\begin{array}{l}
q_{2,1} \\
q_{2,2}
\end{array}\right) .
\end{aligned}
$$


The new cost function becomes

$$
J(\xi, q)=\left(\hat{x}_{3,1}\left(\xi_{3} \mid \xi, q\right)-0.5\right)^{2}+\left(\hat{x}_{3,2}\left(\xi_{3} \mid \xi, q\right)-0.25\right)^{2} .
$$

Using this transformation, we need to impose the constraints

$$
\xi_{i} \geq 0.1, \quad i=1,2,3, \quad \sum_{i=1}^{3} \xi_{i}=1 .
$$

To calculate $\partial \hat{x}_{3}\left(\xi_{3} \mid \xi, q\right) / \partial \xi_{1}$, we solve the vector-valued delay differential equations

$$
\begin{aligned}
& \frac{d y_{(1)}^{(1,2)}\left(s_{2}\right)}{d s_{2}}=\left(\begin{array}{cc}
-2 \hat{x}_{2,2}\left(s_{2}\right) & -2 \hat{x}_{2,1}\left(s_{2}\right) \\
\hat{x}_{2,2}\left(s_{2}\right) & \hat{x}_{2,1}\left(s_{2}\right)
\end{array}\right) y_{(1)}^{(1,2)}\left(s_{2}\right) \\
&+\left(\begin{array}{cc}
0 & \cos \left(\tilde{x}_{2,2}\left(s_{2}\right)\right) \\
\tilde{x}_{2,2}\left(s_{2}\right) & \tilde{x}_{2,1}\left(s_{2}\right)
\end{array}\right) \tilde{y}_{(1)}^{(1,2)}\left(s_{2}\right), \\
& \frac{d y_{(1)}^{(1,3)}\left(s_{3}\right)}{d s_{3}}=\left(\begin{array}{cc}
-2 & 0 \\
0 & -1
\end{array}\right) y_{(1)}^{(1,3)}\left(s_{3}\right)+\left(\begin{array}{cc}
0 & 3 \\
\tilde{x}_{3,2}\left(s_{3}\right) & \tilde{x}_{3,1}\left(s_{3}\right)
\end{array}\right) \tilde{y}_{(1)}^{(1,3)}\left(s_{3}\right),
\end{aligned}
$$

with initial conditions for $y_{(1)}^{(1,2)}\left(s_{2}\right)$ and $y_{(1)}^{(1,3)}\left(s_{3}\right)$ given by

$$
\begin{aligned}
& y_{(1)}^{(1,2)}(0)=\left(\left(\begin{array}{ll}
1 & 0 \\
0 & 1
\end{array}\right)+\left(\begin{array}{cc}
0 & q_{1,1} \\
q_{1,2} & 0
\end{array}\right)\right)\left(\begin{array}{c}
2 \hat{x}_{1,1}\left(\xi_{1}\right) \hat{x}_{1,2}\left(\xi_{1}\right)+\tilde{x}_{1,2}\left(\xi_{1}\right) \\
3 \hat{x}_{1,1}\left(\xi_{1}\right)+4 \tilde{x}_{1,2}\left(\xi_{1}\right)
\end{array}\right), \\
& y_{(1)}^{(1,3)}(0)=\left(\left(\begin{array}{ll}
1 & 0 \\
0 & 1
\end{array}\right)+\left(\begin{array}{cc}
0 & q_{2,1} \\
q_{2,2} & 0
\end{array}\right)\right) y_{(1)}^{(1,2)}\left(\xi_{2}\right) .
\end{aligned}
$$

This gives

$$
\frac{\partial \hat{x}_{3}\left(\xi_{3} \mid \xi, q\right)}{\partial \xi_{1}}=y_{(1)}^{(1,3)}\left(\xi_{3}\right) .
$$

To calculate $\partial \hat{x}_{3}\left(\xi_{3} \mid \xi, q\right) / \partial \xi_{2}$, we solve the vector-valued delay differential equation

$$
\frac{d y_{(1)}^{(2,3)}\left(s_{3}\right)}{d s_{3}}=\left(\begin{array}{cc}
-2 & 0 \\
0 & -1
\end{array}\right) y_{(1)}^{(2,3)}\left(s_{3}\right)+\left(\begin{array}{cc}
0 & 3 \\
\tilde{x}_{3,2}\left(s_{3}\right) & \tilde{x}_{3,1}\left(s_{3}\right)
\end{array}\right) \tilde{y}_{(1)}^{(2,3)}\left(s_{3}\right),
$$

with initial condition for $y_{(1)}^{(2,3)}\left(s_{3}\right)$ given by

$$
y_{(1)}^{(2,3)}(0)=\left(\left(\begin{array}{ll}
1 & 0 \\
0 & 1
\end{array}\right)+\left(\begin{array}{cc}
0 & q_{2,1} \\
q_{2,2} & 0
\end{array}\right)\right)\left(\begin{array}{l}
-2 \hat{x}_{2,1}\left(\xi_{2}\right) \hat{x}_{2,2}\left(\xi_{2}\right)+\sin \left(\tilde{x}_{2,2}\left(\xi_{2}\right)\right) \\
\hat{x}_{2,1}\left(\xi_{2}\right) \hat{x}_{2,2}\left(\xi_{2}\right)+\tilde{x}_{2,1}\left(\xi_{2}\right) \tilde{x}_{2,2}\left(\xi_{2}\right)
\end{array}\right) .
$$

This gives

$$
\frac{\partial \hat{x}_{3}\left(\xi_{3} \mid \xi, q\right)}{\partial \xi_{2}}=y_{(1)}^{(2,3)}\left(\xi_{3}\right)
$$


Furthermore,

$$
\frac{\partial \hat{x}_{3}\left(\xi_{3} \mid \xi, q\right)}{\partial \xi_{3}}=\left(\begin{array}{c}
-2 \hat{x}_{3,1}\left(\xi_{3}\right)+3 \tilde{x}_{3,2}\left(\xi_{3}\right) \\
-\hat{x}_{3,2}\left(\xi_{3}\right)+\tilde{x}_{3,1}\left(\xi_{3}\right) \tilde{x}_{3,2}\left(\xi_{3}\right)
\end{array}\right)
$$

It follows that

$$
\begin{aligned}
\frac{\partial J(\xi, q)}{\partial \xi_{1}}= & \left(2\left(\hat{x}_{3,1}\left(\xi_{3} \mid \xi, q\right)-0.5\right), 2\left(\hat{x}_{3,2}\left(\xi_{3} \mid \xi, q\right)-0.25\right)\right) y_{(1)}^{(1,3)}\left(\xi_{3}\right), \\
\frac{\partial J(\xi, q)}{\partial \xi_{2}}= & \left(2\left(\hat{x}_{3,1}\left(\xi_{3} \mid \xi, q\right)-0.5\right), 2\left(\hat{x}_{3,2}\left(\xi_{3} \mid \xi, q\right)-0.25\right)\right) y_{(1)}^{(2,3)}\left(\xi_{3}\right), \\
\frac{\partial J(\xi, q)}{\partial \xi_{3}}= & \left(2\left(\hat{x}_{3,1}\left(\xi_{3} \mid \xi, q\right)-0.5\right), 2\left(\hat{x}_{3,2}\left(\xi_{3} \mid \xi, q\right)-0.25\right)\right) \\
& \times\left(\begin{array}{c}
-2 \hat{x}_{3,1}\left(\xi_{3}\right)+3 \tilde{x}_{3,2}\left(\xi_{3}\right) \\
-\hat{x}_{3,2}\left(\xi_{3}\right)+\tilde{x}_{3,1}\left(\xi_{3}\right) \tilde{x}_{3,2}\left(\xi_{3}\right)
\end{array}\right) .
\end{aligned}
$$

To calculate $\partial \hat{x}_{3}\left(\xi_{3} \mid \xi, q\right) / \partial q_{1}$, we solve the matrix-valued delay differential equations

$$
\begin{aligned}
\frac{d y_{(2)}^{(1,2)}\left(s_{2}\right)}{d s_{2}}= & \left(\begin{array}{cc}
-2 \hat{x}_{2,2}\left(s_{2}\right) & -2 \hat{x}_{2,1}\left(s_{2}\right) \\
\hat{x}_{2,2}\left(s_{2}\right) & \hat{x}_{2,1}\left(s_{2}\right)
\end{array}\right) y_{(2)}^{(1,2)}\left(s_{2}\right) \\
& +\left(\begin{array}{cc}
0 & \cos \left(\tilde{x}_{2,2}\left(s_{2}\right)\right) \\
\tilde{x}_{2,2}\left(s_{2}\right) & \tilde{x}_{2,1}\left(s_{2}\right)
\end{array}\right) \tilde{y}_{(2)}^{(1,2)}\left(s_{2}\right), \\
\frac{d y_{(2)}^{(1,3)}\left(s_{3}\right)}{d s_{3}}= & \left(\begin{array}{cc}
-2 & 0 \\
0 & -1
\end{array}\right) y_{(2)}^{(1,3)}\left(s_{3}\right)+\left(\begin{array}{cc}
0 & 3 \\
\tilde{x}_{3,2}\left(s_{3}\right) & \tilde{x}_{3,1}\left(s_{3}\right)
\end{array}\right) \tilde{y}_{(2)}^{(1,3)}\left(s_{3}\right),
\end{aligned}
$$

with initial conditions for $y_{(2)}^{(1,2)}\left(s_{2}\right)$ and $y_{(2)}^{(1,3)}\left(s_{2}\right)$ given by

$$
\begin{aligned}
& y_{(2)}^{(1,2)}(0)=\left(\begin{array}{cc}
\hat{x}_{1,2}\left(\xi_{1}\right) & 0 \\
0 & \hat{x}_{1,1}\left(\xi_{1}\right)
\end{array}\right) \\
& y_{(2)}^{(1,3)}(0)=\left(\left(\begin{array}{ll}
1 & 0 \\
0 & 1
\end{array}\right)+\left(\begin{array}{cc}
0 & q_{2,1} \\
q_{2,2} & 0
\end{array}\right)\right) y_{(2)}^{(1,2)}\left(\xi_{2}\right) .
\end{aligned}
$$

This gives

$$
\frac{\partial \hat{x}_{3}\left(\xi_{3} \mid \xi, q\right)}{\partial q_{1}}=y_{(2)}^{(1,3)}\left(\xi_{3}\right)
$$

To calculate $\partial \hat{x}_{3}\left(\xi_{3} \mid \xi, q\right) / \partial q_{2}$, we solve the matrix-valued delay differential equation

$$
\frac{d y_{(2)}^{(2,3)}\left(s_{3}\right)}{d s_{3}}=\left(\begin{array}{cc}
-2 & 0 \\
0 & -1
\end{array}\right) y_{(2)}^{(2,3)}\left(s_{3}\right)+\left(\begin{array}{cc}
0 & 3 \\
\tilde{x}_{3,2}\left(s_{3}\right) & \tilde{x}_{3,1}\left(s_{3}\right)
\end{array}\right) \tilde{y}_{(2)}^{(2,3)}\left(s_{3}\right),
$$

with initial condition for $y_{(2)}^{(2,3)}\left(s_{3}\right)$ given by

$$
y_{(2)}^{(2,3)}(0)=\left(\begin{array}{cc}
\hat{x}_{2,2}\left(\xi_{2}\right) & 0 \\
0 & \hat{x}_{2,1}\left(\xi_{2}\right)
\end{array}\right) .
$$




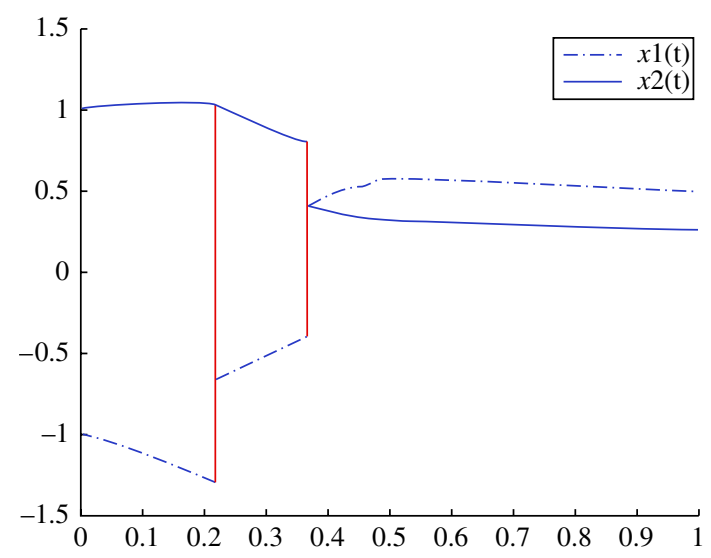

FIGURE 1. The state of the example.

This gives

$$
\frac{\partial \hat{x}_{3}\left(\xi_{3} \mid \xi, q\right)}{\partial q_{2}}=y_{(2)}^{(2,3)}\left(\xi_{3}\right)
$$

Therefore,

$$
\begin{aligned}
& \frac{\partial J(\xi, q)}{\partial q_{1}}=\left(2\left(\hat{x}_{3,1}\left(\xi_{3} \mid \xi, q\right)-0.5\right), 2\left(\hat{x}_{3,2}\left(\xi_{3} \mid \xi, q\right)-0.25\right)\right) y_{(2)}^{(1,3)}\left(\xi_{3}\right), \\
& \frac{\partial J(\xi, q)}{\partial q_{2}}=\left(2\left(\hat{x}_{3,1}\left(\xi_{3} \mid \xi, q\right)-0.5\right), 2\left(\hat{x}_{3,2}\left(\xi_{3} \mid \xi, q\right)-0.25\right)\right) y_{(2)}^{(2,3)}\left(\xi_{3}\right) .
\end{aligned}
$$

We use the algorithm developed in Section 3 to solve this problem. The results obtained are:

$$
\xi_{1}=0.2184, \quad \xi_{2}=0.1500, \quad \xi_{3}=0.6316,
$$

yielding $\tau_{1}=0.2184, \tau_{2}=0.3784, \tau_{3}=1.0000$;

$$
q_{1}=(0.5998,0)^{T}, \quad q_{2}=(1,1)^{T} ;
$$

and the optimal cost is 0.00014853 . The behavior of the state variable is depicted in Figure 1. The behavior of the state variable at the optimal switching times is as follows. At the first optimal switching time $\tau_{1}$, the state variable changes from

$$
x\left(\tau_{1}^{-}\right)=\left(\begin{array}{c}
-1.287 \\
1.006
\end{array}\right) \quad \text { to } \quad x\left(\tau_{1}^{+}\right)=\left(\begin{array}{c}
-0.684 \\
1.006
\end{array}\right),
$$

due to the impulsive control law (4.1). At the second optimal switching time $\tau_{2}$, the state variable changes from

$$
x\left(\tau_{2}^{-}\right)=\left(\begin{array}{c}
-0.393 \\
0.774
\end{array}\right) \quad \text { to } \quad x\left(\tau_{2}^{+}\right)=\left(\begin{array}{c}
0.381 \\
0.381
\end{array}\right),
$$

also due to (4.1). 


\section{Conclusion and suggestions for further study}

A computational method has been developed for solving a class of optimal control problems governed by switched and impulsive systems with time delay. A numerical example solved using the proposed method illustrates its applicability. Potential future research is the development of an effective computational method for solving optimal control problems governed by switched impulsive systems with time delay, which includes also time-varying controls in the state equations.

\section{Acknowledgements}

This work is partially supported by the Natural Science Foundation Project of CQ CSTC (No. CSTC, 2010BB2090), Education Commission Project Research Foundation of Chongqing (No. J110617), P. R. China, and Ministry of Education of the People's Republic of China.

\section{References}

[1] R. Gao, X. Liu and J. Yang, "On optimal control of a class of impulsive switching systems with terminal states constraints”, Nonlinear Anal. Theory Methods Appl. 73 (2010) 1940-1951; doi:10.1016/j.na.2010.05.013.

[2] Z. H. Guan, D. J. Hill and X. Shen, "On hybrid impulsive and switching systems and application to nonlinear control", IEEE Trans. Automat. Control 50 (2005) 1058-1062; doi:10.1109/TAC.2005.851462.

[3] J. Hu, H. Wang, X. Liu and B. Liu, "Optimization problems for switched systems with impulsive control”, J. Control Theory Appl. 3 (2005) 93-100; doi:10.1007/s11768-005-0067-5.

[4] C. Jiang, K. L. Teo, R. Loxton and G. R. Duan, "A neighboring extremal solution for an optimal switched impulsive control problem", J. Indust. Manag. Optim. 8 (2012) 591-609; doi:10.3934/jimo.2012.8.591.

[5] C. Y. Kaya and J. L. Noakes, "Computational method for time-optimal switching control", J. Optim. Theory Appl. 117 (2003) 69-92; doi:10.1023/A:1023600422807.

[6] R. Li, Z. G. Feng, K. L. Teo and G. R. Duan, "Optimal piecewise state feedback control for impulsive switched systems”, Math. Comput. Modelling 48 (2008) 468-479; doi:10.1016/j.mcm.2007.06.028.

[7] Q. Lin, R. Loxton, K. L. Teo and Y. H. Wu, "A new computational method for optimizing nonlinear impulsive systems", Dyn. Contin. Discrete Impuls. Syst. Ser. B 18 (2011) 59-76.

[8] C. Y. Liu, Z. H. Gong, E. M. Feng and H. C. Yin, "Modelling and optimal control for nonlinear multistage dynamical system of microbial fed-batch culture", J. Indust. Manag. Optim. 5 (2009) 835-850; doi:10.3934/jimo.2009.5.835.

[9] G. Rui, "Towards optimal control problems of hybrid impulsive and switching systems with free terminal states", Chinese J. Electronics 19 (2010) 557-562.

[10] E. I. Verriest, "Regularization method for optimally switched and impulsive systems with biomedical applications", in: Proc. 42nd IEEE Conf. on Decision and Control, 2003, 2156-2161; doi:10.1109/CDC.2003.1272937.

[11] E. I. Verriest, "Optimal control for switched point delay systems with refractory period", in: Proc. 16th IFAC World Congress, 2005.

[12] E. I. Verriest, "Optimal control for switched distributed delay systems with refractory period", in: Proc. 44th IEEE Conf. on Decision and Control, 2005, 1421-1426; doi:10.1109/CDC.2005.1582358.

[13] E. I. Verriest, F. Delmotte and M. Egerstedt, "Optimal impulsive control for point delay systems with refractory period”, in: Proc. 5th IFAC Workshop on Time Delay Systems, 2004. 
[14] C. Wu, K. L. Teo, R. Li and Y. Zhao, "Optimal control of switched systems with time delay", Appl. Math. Lett. 19 (2006) 1062-1067; doi:10.1016/j.aml.2005.11.018.

[15] C. Z. Wu, K. L. Teo and R. Volker, "Optimal control of switched system with time delay detection of switching signal", in: Numerical linear algebra in signals, systems and control, Volume 80 of Lecture Notes in Electrical Engineering (eds P. Van Dooren et al.), (Springer, Dordrecht, 2001), 467-478; doi:10.1007/978-94-007-0602-6_21.

[16] Y. Yin, J. Zhao and Y. Liu, "H-infinity control for switched and impulsive singular systems", J. Control Theory Appl. 6 (2008) 86-92; doi:10.1007/s11768-008-6140-0. 